\title{
Minimally invasive spinal anesthesia for cesarean section in maternal anticoagulation therapy: a randomized controlled trial
}

Dan Huang ${ }^{\dagger}$, Linjie Zhu ${ }^{\dagger}$, Jie Chen ${ }^{*}$ and Jie Zhou ${ }^{*}$ (D)

\begin{abstract}
Background: Anticoagulant therapy during pregnancy is widely used due to the increasing awareness of maternal hypercoagulability. Few studies have reported the use of minimally invasive spinal anesthesia in these parturients. The objective of this study was to evaluate the safety and feasibility of minimally invasive spinal anesthesia in parturients with anticoagulation therapy undergoing cesarean section.

Methods: This was a randomized, controlled study conducted in 239 parturients using anticoagulants and undergoing selective cesarean section. 37 parturients withdrew, and finally parturients received spinal anesthesia using 27gauge pen type fine spinal needles (experimental group, $n=110$ ) and 22gauge traditional spinal needles (control group, $n=92$ ). The primary efficacy outcomes included low back pain (LBP) and postdural puncture headache (PDPH) after delivery. Secondary efficacy outcomes included visual analogue scale during subarachnoid puncture (VASdural), difference between visual analogue scale (VAS) during peripheral venipuncture and VASdural ( $\triangle$ VAS), VAS of back puncture point 24,48 and $72 \mathrm{~h}$ after operation (VASdural-24 $\mathrm{h}$, VASdural-48 $\mathrm{h}$ and VASdural-72 $\mathrm{h}$, respectively), maternal satisfaction and hospitalization stay.
\end{abstract}

Results: No parturient had PDPH and was suspected with spinal or intracranial haematoma in two groups. There was no significant difference in VASlbp-24 h, VASlbp-48 h and VASlbp-72 $\mathrm{h}(P=0.056 ; P=0.813 ; P=0.189$, respectively) between two groups. In experimental group, VASdural $(P=0.017), \triangle V A S(P=0.001)$ and VASdural-24 $\mathrm{h}$ $(P<0.0001)$ were lower, whereas maternal satisfaction was higher $(P=0.046)$. There was no significant difference in VASdural-48 h, VASdural-72 h, urination function, strength recovery and hospitalization stay $(P=0.069 ; P=0.667$; $P=0.105 ; P=0.133 ; P=0.754$, respectively) between the two groups.

Conclusions: Minimally invasive spinal anesthesia provided lower VASdural, VASdrual-24 $\mathrm{h}$ and a higher maternal satisfaction. Hence, it is considered as a safe, reliable and reasonable option for cesarean section parturients during maternal anticoagulation therapy with normal platelet count and coagulation time.

Trial registration: This study was registered at www.ClinicalTrials.gov at November 11th, 2016 (NCT02987192).

Keywords: Minimally invasive, Low back pain, Postdural puncture headache, Spinal anesthesia, Cesarean section, Anticoagulants

\footnotetext{
*Correspondence: 13611906592@163.com; 13601693227@163.com

${ }^{\dagger}$ Dan Huang and Linjie Zhu contributed equally to this work.

Department of Anesthesiology, Renji Hospital, School of Medicine, Shanghai

Jiaotong University, Shanghai 200127, China
}

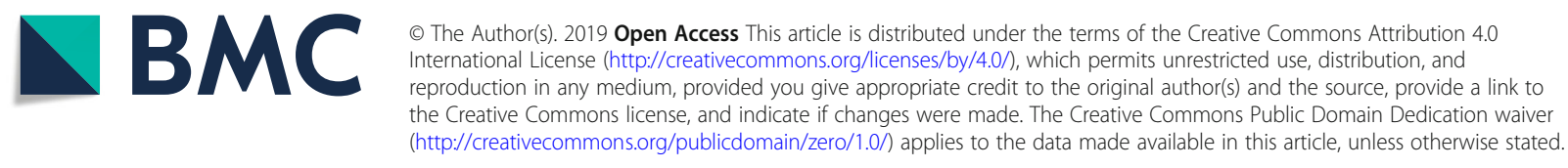




\section{Background}

According to the World Health Organization (WHO) Global Survey on Maternal and Perinatal Health in 2010, Chinese health facilities has the highest cesarean section rate (CSR) (46.2\%) in Asia (27.3\%) [1], and the CSR had risen far in excess of the optimal 15\% recommended by WHO [1, 2]. According to a recent study, the overall annual rate of cesarean deliveries between 2008 and 2014 was increased in China, i.e., up to $34.9 \%$ [3]. With the implementation of second child policy, the ratio still remained high. Intravertebral anesthesia is the most commonly used method for parturients undergoing cesarean section. Traditional intravertebral anesthesia may cause postdural puncture headache (PDPH) and low back pain.

PDPH may require epidural blood patching, resulting in prolonged hospital stay and emergency visits after discharge [4, 5]. Previous study has shown that the incidence of PDPH was about 7 to 30\% [6]. Decrease of intracranial pressure due to cerebrospinal fluid (CSF) leakage from the subarachnoid space is currently the leading cause of PDPH [5]. The PDPH, which causes significant morbidity in obstetric patients, has higher incidence because of the increased CSF pressure related to pregnancy, dehydration, blood loss, postpartum diuresis, hormonal imbalance, high serum estrogen levels, and increased peridural pressure [7].

Low back pain (LBP) commonly occurs during late pregnancy and also after delivery. Several research studies revealed that at least half of the pregnant women is affected by LBP $[8,9]$. Persistence of LBP for 6 months after delivery has been reported in 5 to $40 \%$ of patients [10]. A retrospective study of 40,057 women demonstrated that cesarean delivery and epidural anesthesia might increase the risk of subsequent chronic LBP [11]. The paraspinal muscular relaxtion with stretching of spinal ligaments and/or localized tissue trauma are accepted as the pathophysiological factors of LBP after puncture.

The concept of minimally invasive spinal anesthesia has been put forwarded due to its appearance of pen type lumbar puncture needle and unceasing enhancement of piercing technology. It refers to puncturing with 27 gauge pen type fine needle to reduce the damage of supraspinous, interspinous and yellow ligament by puncture needle.

However, there are relative contraindications to spinal anesthesia, one of which is the use of anticoagulants. Anticoagulant therapy during pregnancy is widely used due to the increasing awareness of maternal hypercoagulability [12]. The pregnant women are forced to accept general anesthesia in order to avoid epidural hematoma and lose the experience of baby birth moment. Minimally invasive spinal anesthesia happens to solve this problem, reducing the side effects without increasing the risk of spinal anesthesia for maternal anticoagulation therapy.
We therefore performed a randomized, controlled trial in parturients undergoing anticoagulation therapy to evaluate the safety and feasibility of minimally invasive spinal anesthesia. We also investigated the use of 27gauge pen type fine spinal needles and 22 gauge traditional spinal needles for spinal anesthesia on PDPH and LBP in parturients with anticoagulation therapy undergoing cesarean section.

\section{Methods}

\section{Research design}

This was a prospective randomized controlled study conducted in accordance with the ethics and data protection of Chinese regulations. The protocol was approved by the ethics committee of Renji hospital, School of Medicine, Shanghai Jiaotong University. Written informed consent was obtained from all the parturients before randomization in this prospective, parallel-arm, randomized clinical trial. This trial was a single-site study conducted at the Shanghai Renji hospital. This study was registered at www.ClinicalTrials.gov (NCT02987192).

\section{Participants}

Consecutive parturients aged $\geq 18$ years with singleton pregnancy puerpera who were using anticoagulation medications and undergoing selective cesarean section were eligible for inclusion between November 2016 and December 2017. Only parturients with the American Society of Anesthesiologists physical status between I and II were included. Exclusion criteria were as follows: parturients with a history of migraine headache, previous history of $\mathrm{PDPH}$, platelet count lower than $70^{*} 10^{\wedge} 9$, International Normalized Ratio (INR) > 1.5, severe cardiopulmonary insufficiency, infection of skin or subcutaneous tissue at the puncture site, lumbar or spinal cord disease, increased intracranial pressure, weight $>100 \mathrm{~kg}$ or $<50 \mathrm{~kg}$ and height $>165 \mathrm{~cm}$ or $<155 \mathrm{~cm}$.

\section{Randomization}

Parturients were randomized in 1:1 ratio to the two study groups by means of a central telephone system to ensure concealed allocation. Randomization was performed using a computer-generated randomization sequence with randomly permuted blocks of 4 or 6 . Patient caregivers and investigators collecting the data remained unaware of study-group assignments.

\section{Interventions}

Electrocardiogram, blood pressure, pulse oxygen saturation, and heart rate were measured after the parturients entered the operation room. A 20gauge indwelling needle was placed into the peripheral vein. Thromboelastogram, platelet count and coagulation time were detected. All parturients were hydrated with $8 \mathrm{~mL} / \mathrm{kg}$ body weight Ringer's lactated solution before spinal anesthesia. Spinal 
anesthesia was performed with a standardized technique. All parturients received spinal anesthesia by an experienced anesthetist. The parturients in the experimental group received spinal anesthesia using 27gauge pen type fine needles (HaiSheng, China), and control group parturients received spinal anesthesia using 22gauge traditional needles (HaiSheng, China). Lumbar puncture was performed in the intervertebral space of $\mathrm{L} 2-3$ by placing the parturients in the right lateral decubitus position. The parturients were instructed to remain flexed and not to move their heads during the insertion of the needle. Spinal needle was inserted through the longitudinal dural fiber using a midline approach at L2-3. After verifying the free flow of CSF through the needle tip, $0.75 \%$ ropivacaine $2 \mathrm{~mL}$ was injected within $5 \mathrm{~s}$.

Two consecutive episodes of hypotension (defined as systolic blood pressure less than $80 \%$ of baseline) were treated with a "rescue" intravenous bolus of $100 \mu \mathrm{g}$ phenylephrine and volume expansion. When the skin closure begined, intravenous Patient-Controlled Analgesia (PCA) pump was commenced to infuse $100 \mathrm{~mL}$ mixture of sufentanil $(0.045 \mu \mathrm{g} / \mathrm{kg} / \mathrm{h})$, flurbiprofen $(45 \mu \mathrm{g} / \mathrm{kg} / \mathrm{h})$, and saline at a rate of $2 \mathrm{~mL} / \mathrm{h}$ using an analgesia pump (Apon, China). PCA administration was continued until $48 \mathrm{~h}$ postoperatively in the two groups.

\section{Outcome measures}

The primary efficacy outcomes calculated were LBP and PDPH after puncture. LBP after puncture was defined as the continuous pain and tenderness over the lumbar area around the spinal needle insertion and was recorded using visual analogue scale (VASlbp) on the postoperative days [13]. Pain was defined on a scale of 0 as the absence of pain, and 7-10 as severe pain (disabling; unable to perform daily activities). PDPH was defined according to the International Headache Society Classifications, bifrontal or occipital region headache that remained worse in the upright position and relieved with supine posture and headache within 5 days after dural puncture [14]. Other types of headaches were considered nonspecific and were excluded from the study. The intensity of PDPH was also recorded using visual analogue scale (VASPDPH) on postoperative day 1. Secondary efficacy outcomes included were visual analogue scale during subarachnoid puncture (VASdural), difference between VAS during peripheral venipuncture (VASperipheral) and VASdural ( $\triangle \mathrm{VAS})$, VAS of back puncture point 24,48 and $72 \mathrm{~h}$ after operation (VAS24h, VAS48h and VAS72h), maternal satisfaction and hospitalization days. The safety outcome was the incidence of hypotension after anesthesia in the operation room. The dosage and withdrawal time of anticoagulants were also recorded.

\section{Sample size calculation}

Sample size calculation was based on the data from previous studies. The incidence of PDPH in 22gauge needle was reported as 34\% [6] and LBP after caesarean delivery with spinal anesthesia was $31.8 \%$ [11], respectively. By assuming a fall by half of the incidences, we calculated that a sample size of 82 parturients per group would be required to achieve a power of $90 \%$ with a two-sized $\alpha$ risk of 0.05 , which was calculated using PASS software, version 15.0(NSCC, USA).

\section{Statistical analysis}

Categorical data were described as frequencies and proportions and were analyzed using Chi-square test. Kolmogorov-Smirnov test was used to examine the normality of distribution of continuous outcomes. Normally distributed continuous variables were described as mean \pm standard deviation $(\mathrm{SD})$ and were analyzed using Student's $t$ test, whereas non-normally distributed continuous variables were presented as median \pm interquartile range (IQR) and were performed with the MannWhitney U test. Significance was set at 0.05 level. Statistical analyses were performed using GraphPad Prism software, version 6.01(GraphPad Software, Inc. USA).

\section{Results}

Demographic, physiologic and surgical characteristics

A total of 239 parturients were randomly assigned to the experimental group $(n=120)$ or control group $(n=119)$. 37 participants withdrew during our study period; $84.5 \%$ of 239 parturients were analyzed in our study (Fig. 1). There were 10 withdraws in the experiment group: 1 subject was transferred to the intensive care unit (ICU) immediately post-operatively because of overmuch blood loss during operation, and other 9 subjects met exclusion criteria. 18 subjects in the control group met exclusion criteria and other 9 subjects decided not to participate in the study further after operation without specific reason. Thus, the final numbers of participants were 110 in experiment group and 92 in control group. Both patient characteristics and surgical characteristics were similar in the two groups. Birthweight was also similar between the groups and Apgar score of each neonate at $1 \mathrm{~min}$ and 5 min was 10 (Table 1).

\section{Use of anticoagulants and coagulation function}

Maternal anticoagulation therapy was shown in Table 2 and Table 3. Daily dosage of low molecular weight heparin (LMWH) was $4100 \mathrm{U}$. There was no significant difference in the dose and time of anticoagulant between the two groups. Aspirin was the most common anticoagulant medication and alone or duplex anticoagulation medications favored more. There was no significant difference in thromboelastogram, platelet count and coagulation time 


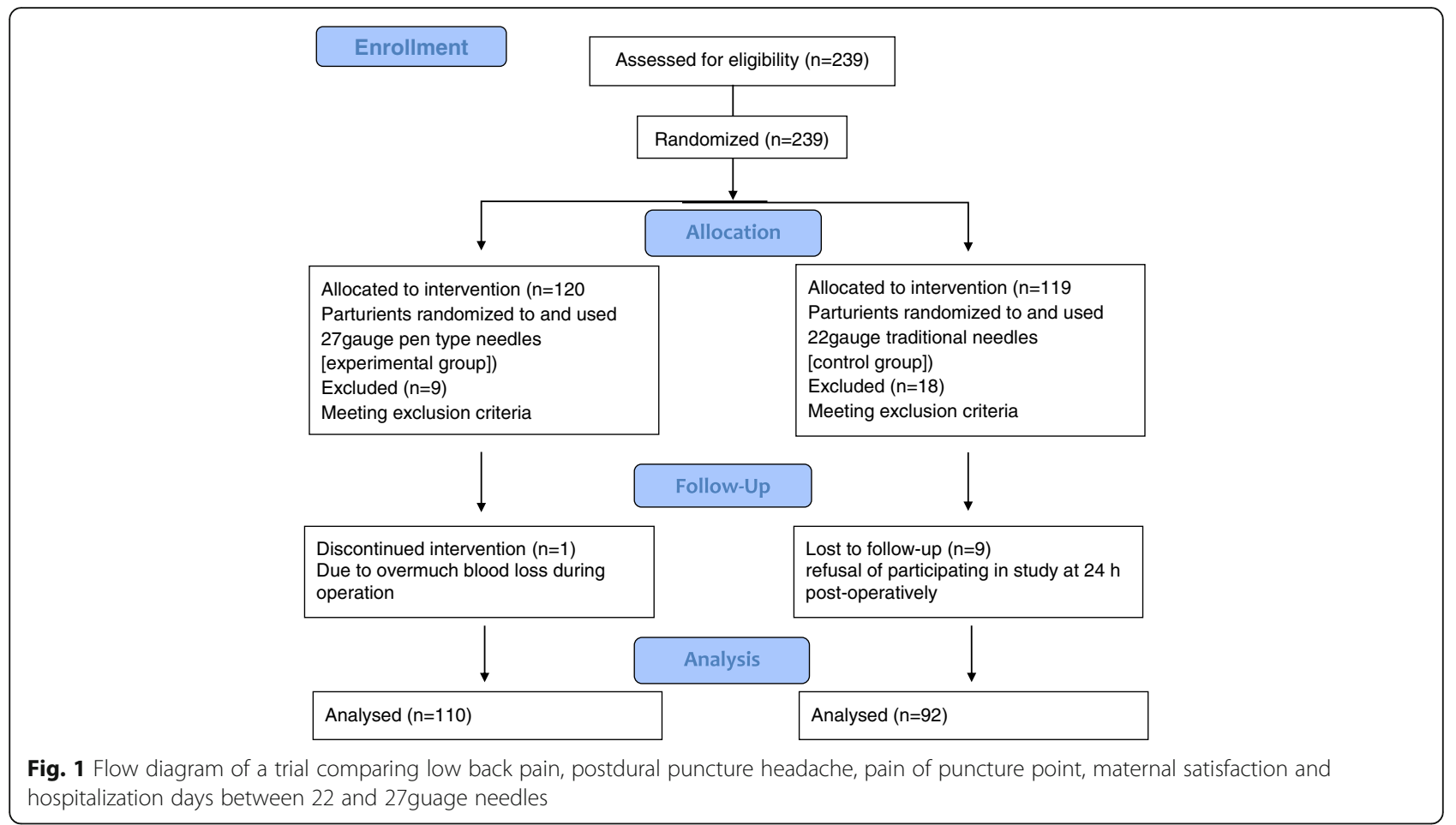

between the two groups. D-Dimer and $\alpha$ angle in the two groups were higher than normal (Table 4). None of the parturients was suspected of spinal or intracranial hematoma.

\section{Primary and secondary efficacy outcomes}

In the primary efficacy outcomes, no parturient suffered from PDPH and no significant difference in VASlbp-24 $\mathrm{h}$, VASlbp- $48 \mathrm{~h}$ and VASlbp- $72 \mathrm{~h}$ between the two groups was observed $(P=0.056 ; P=0.813 ; P=0.189$, respectively). In the secondary efficacy outcomes, VASdural, $\triangle$ VAS and VAS24h in the experimental group were lower than control group (VASdural median 3.0 vs 4.0, $P=0.017$; $\triangle$ VAS median 0.0 vs $1.0, P=0.001$; VASdural-24h median 0.0 vs $1.0, P<0.0001)$. Maternal satisfaction in experimental group was higher than control group (98.2\% vs $91.3 \%, P=0.046)$. No significant difference in VASdural-48 $\mathrm{h}$, VASdural-72 $\mathrm{h}$ and hospitalization stay was observed between the two groups $(P=0.069 ; P=0.667$; $P=0.754$, respectively).

No differences in the feelings during puncture, puncture time, puncture point bleeding, inserting depth of puncture needle and the time of analgesia block level that reached T6 were observed between the two groups ( $P=0.062 ; P=0.644 ; P=0.740 ; P=0.073$, respectively). Parturients in the two groups had similar incidence of hypotension and received similar volume of vasopressor $(P=0.123 ; P=0.073$, respectively). There was no significant

Table 1 Baseline Parturients and Surgical Characteristics

\begin{tabular}{llll}
\hline Characteristics & Experimental group $(n=110)$ & Control group $(n=92)$ & $P$ value \\
\hline Age, median (IQR), y & $32.0(29.0-35.0)$ & $32.0(28.0-35.0)$ & $161.0(158.0-165.0)$ \\
Height, median (IQR), cm & $160.0(158.0-163.0)$ & $26.5(3.3)$ & .474 \\
Body mass index, mean(SD) & $26.6(3.7)$ & $38.0(37.0-38.0)$ & .527 \\
Gestational weeks, median (IQR) & $38.0(37.0-38.0)$ & $38(41.3)$ & .869 \\
ASA status I, $n$ (\%) & $50(45.0)$ & $12(13.0)$ & .643 \\
History of spinal anesthesia, $n$ (\%) & $16(14.5)$ & $41.5(35.0-48.0)$ & .572 \\
Operation time, median (IQR), min & $41.0(33.0-50.0)$ & $7.0(6.0-9.0)$ & .839 \\
Induction-to-delivery time, median (IQR), min & $6.0(5.0-10.0)$ & $3150.0(2910.0-3410.0)$ \\
Newborn weight, median (IQR), g & $3130.0(2830.0-3375.0)$ & $3.0(3.0-3.0)$ \\
Hospital stay, median (IQR), d & $3.0(3.0-4.0)$ & .620
\end{tabular}

SD Standard Deviation, IQR Interquartile Range, ASA American Society of Anesthesiologists 
Table 2 Dosage and Withdrawal Time of Anticoagulant

\begin{tabular}{llll}
\hline Variables & Experimental group $(n=110)$ & Control group $(n=92)$ & $P$ value \\
\hline Daily dosage of aspirin, median (IQR), mg & $25.0(25.0-50.0)$ & $25.0(25.0-50.0)$ & $3.0(1.0-5.0)$ \\
Withdrawal time of aspirin, median (IQR), d & $4.0(0.3-5)$ & $88(95.7)$ & .135 \\
Number of aspirin users, $\mathrm{n}(\%)$ & $104(94.5)$ & $12.5(11.0-15.0)$ \\
Withdrawal time of LMWH, median (IQR), h & $11.0(8.8-13.0)$ & $44(47.8)$ & .705 \\
Number of LMWH users, $n$ (\%) & $66(60.0)$ & $0.3(0.3-0.6)$ & .096 \\
Daily dosage of Salvia miltiorrhiza, median (IQR), g & $0.3(0.3-0.6)$ & $0.0(0.0-2.0)$ & .091 \\
Withdrawal time of Salvia miltiorrhiza, median (IQR), d & $1.0(0.0-1.0)$ & $14(15.2)$ & .057 \\
Number of Salvia miltiorrhiza users, $n(\%)$ & $14(12.7)$ & & .686 \\
\hline
\end{tabular}

IQR Interquartile Range, LMWH Low Molecular Weight Heparin

difference in urination function, strength recovery and remedy for analgesia after operation between the two groups $(P=0.105 ; P=0.133 ; P=0.344$, respectively) (Table 5).

\section{Discussion}

Our research indicated that minimally invasive spinal anesthesia was a reliable and reasonable option for cesarean section in maternal anticoagulation therapy with normal platelet count and coagulation time. Previous literature concluded that the anticoagulant treatment continues to be contraindicated to spinal/epidural anesthesia [15]. Spinal-epidural hematoma during spinal anesthesia was described in 1/200000-250,000. Spinal or intracranial hematoma is a rare but a severe complication [16]. Traumatic puncture was assumed to be the risk factor for the development of this kind of hematoma. Anticoagulant treatments increase the risk of spinal hematoma to $1 / 40000$ in patients undergoing anticoagulation treatment [17]. To prevent spinal hematoma, neuraxial anesthesia is prohibited when the anticoagulant treatment is used as curative dose during delivery. These conclusions refer to the relatively coarse technology and traditional lumbar puncture needle. In our study, there was no significant difference in the puncture point bleeding between the two groups. Defecation function, lower limb strength and sensation of all parturients in our study were normal. We inferred that no parturient was suspected of spinal or intracranial hematoma. Minimally invasive spinal anesthesia in our research was considered as safe and effective approach for maternal anticoagulation

Table 3 Use of Anticoagulants

\begin{tabular}{llllrr}
\hline $\begin{array}{l}\text { Types of } \\
\text { drugs }\end{array}$ & $\begin{array}{l}\text { Alone } \\
\text { medication }\end{array}$ & $\begin{array}{l}\text { Duplex } \\
\text { medication }\end{array}$ & $\begin{array}{l}\text { Triple } \\
\text { medication }\end{array}$ & Total, $n$ & $\%$ \\
\hline Aspirin & 82 & 92 & 18 & 192 & 58.2 \\
LMWH & 8 & 84 & 18 & 110 & 33.3 \\
Salvia Miltiorrhiza & 2 & 8 & 18 & 28 & 8.5 \\
Total, $n$ & 92 & 92 & 18 & & \\
$\%$ & 45.5 & 45.5 & 8.9 & & \\
\hline
\end{tabular}

LMWH Low Molecular Weight Heparin due to smaller caliber of puncture needle and lesser damage of intraspinal blood vessel.

No parturient had suffered from PDPH and no significant difference in PDPH using 22 or 27gauge needles was revealed in our observation. Smaller gauge needles were recommended for spinal anesthesia to reduce the incidence of PDPH. Kim et al. reported no significant difference in the cause of PDPH by using 23 and 25 gauge needles in spinal anesthesia [18], which was consistent with our study findings. However, our result differed from the conclusion by Lambert et al. that compared with 26gauge Quincke needle, the incidence of PDPH using a 27gauge Quincke needle was significantly reduced [19]. Previous studies have shown that older people have a lower incidence of PDPH than younger people, which may be related to a decrease in pain perception or pain response in the elderly $[20,21]$. Age is likely to be an important factor in the pathogenesis of PDPH. However, it was different with our result as our study subjects only included young women subjects. A successful initial lumbar puncture and avoiding local muscle-ligamentous trauma caused by repeated punctures due to the clinician's technique can be considered the cause of difference.

Our data demonstrated that there was no significant difference in LBP using either 22 or 27gauge needles. Whereas, we found that the procedure of minimally invasive spinal anesthesia provided a lower VAS during subarachnoid puncture, a lower VAS of back puncture point at $24 \mathrm{~h}$ after operation and a higher maternal satisfaction. However, the conclusions drawn by previous studies showed discrepancies. Lowery et al. reported that $11 \%$ of the oncologic patients were aged between 2 and 17 years, and had LBP after lumbar puncture following the use of a 22gauge cutting Quincke needle [6]. In contrast, Kim M and Yee $\mathrm{K}$, respectively reported no significant difference in pain score in 1-3 days after puncturing with different needles $[18,22]$. Our positive result on the advantage of the use of 27 gauge puncture needles may be due to the reduction of local muscle-ligamentous trauma associated with needle insertion. It was noteworthy that although effective analgesia, muscular relaxation, immobility, 
Table 4 Thromboelastogram, Platelet count and Coagulation Time

\begin{tabular}{llll}
\hline Variables & Experimental group $(n=110)$ & Control group $(n=92)$ & $P$ value \\
\hline D-Dimer, median (IQR) & $0.7(0.4-0.9)^{\mathrm{a}}$ & $0.6(0.4-1.0)^{\mathrm{a}}$ & .607 \\
FDP, median (IQR) & $5.6(3.5-11.0)$ & $6.9(3.8-9.3)$ & $14.5(12.5-16.9)$ \\
TT, median (IQR) & $16.4(12.9-17.4)$ & $27.6(25.1-29.0)$ & .627 \\
APTT, median (IQR) & $26.7(24.8-28.1)$ & $10.1(9.4-11.5)$ & .055 \\
PT, median (IQR) & $10.7(9.4-11.4)$ & $4.4(3.9-4.9)$ & .097 \\
Fg, median (IQR) & $4.3(3.9-4.9)$ & $0.9(0.9-1.0)$ & .512 \\
INR, median (IQR) & $1.0(0.9-1.0)$ & $194.5(175.0-211.0)$ & .449 \\
PLT^9, median (IQR) & $198.0(150.0-259.0)$ & $6.9(6.5-7.4)$ & .484 \\
Rtime, median (IQR) & $6.6(5.9-7.3)$ & $1.6(1.5-1.8)$ & .413 \\
Ktime, median (IQR) & $1.6(1.4-1.8)$ & $72.6(71.0-73.6)^{\mathrm{a}}$ & .090 \\
a angle, median (IQR) & $73.5(70.7-75.0)^{\mathrm{a}}$ & $69.6(69.1-70.0)$ & .053 \\
MA, median (IQR) & $69.4(69.1-70.0)$ & .054 \\
\hline
\end{tabular}

${ }^{\mathrm{a} D}$-Dimer and a angle in two groups were higher than normal

IQR Interquartile Range, FDP Fibrinogen Degradation Product, $T$ Thrombin Time, APTT Activated Partial Thromboplastin Time, PT Prothrombin Time, Fg Fibrinogen, INR International Normalized Ratio, PLT Platelets, MA Maximum Amplitude

Table 5 Primary and Secondary Endpoints

\begin{tabular}{|c|c|c|c|}
\hline Variables & Experimental group $(n=110)$ & Control group $(n=92)$ & $P$ \\
\hline VASIbp-24 h, median (IQR) & $3.0(2.0-4.0)$ & $3.0(3.0-4.0)$ & .056 \\
\hline VASIbp-48 h, median (IQR) & $2.0(1.0-3.0)$ & $2.0(1.0-3.0)$ & .813 \\
\hline VASIbp-72 h, median (IQR) & $1.0(0.0-1.0)$ & $1.0(0.0-1.0)$ & .189 \\
\hline VASperipheral, median (IQR) & $3.0(2.0-5.0)$ & $3.0(3.0-4.0)$ & .769 \\
\hline VASdural, median (IQR) & $3.0(1.0-6.0)$ & $4.0(3.0-6.0)$ & .017 \\
\hline$\triangle \mathrm{VAS}$, median (IQR) & $0.0(-1.0-1.0)$ & $1.0(0.0-2.0)$ & .001 \\
\hline VASdural-24 h, median (IQR) & $0.0(0.0-1.0)$ & $1.0(1.0-1.0)$ & $<.0001$ \\
\hline VASdural-48 h, median (IQR) & $0.0(0.0-0.0)$ & $0.0(0.0-1.0)$ & .069 \\
\hline VASdural-72 h, median (IQR) & $0.0(0.0-0.0)$ & $0.0(0.0-0.0)$ & .667 \\
\hline maternal satisfaction (satisfaction, \%) & 108(98.2) & $84(91.3)$ & .046 \\
\hline Puncture time, median (IQR), s & $40.0(22.0-60.0)$ & $60.0(30.0-68.0)$ & .062 \\
\hline Puncture point bleeding (bleeding, \%) & $10(9.1)$ & $11(12.0)$ & .644 \\
\hline Inserting depth of puncture needle, median (IQR), cm & $5.6(5.5-6.0)$ & $6.0(5.3-6.0)$ & .740 \\
\hline Time of analgesia block level reached T6, median (IQR), min & $6.0(6.0-8.0)$ & $8.0(6.0-8.0)$ & .073 \\
\hline Incidence of hypotension, $n$ (\%) & $60(54.5)$ & $40(43.4)$ & .123 \\
\hline Rescue phenylephrine required, median (IQR), $\mu \mathrm{g}$ & 100.0(100.0-200.0) & 100.0(100.0-175.0) & .073 \\
\hline Rescue intraoperative intravenous anesthesia required, $n(\%)$ & $8(7.3)$ & 10(10.9) & .459 \\
\hline Nausea or vomiting, $n$ (\%) & 12(10.9) & $8(8.7)$ & .644 \\
\hline Postoperative self-urination time, median (IQR), $\mathrm{h}$ & 18.0(15.0-20.0) & 19.0(11.0-21.0) & .105 \\
\hline Recovery of lower limb muscle strength, median (IQR), h & $4.0(3.0-5.0)$ & $4.0(4.0-6.0)$ & .133 \\
\hline First remedy for analgesia, median (IQR), h & $5.0(2.0-7.0)$ & $4.0(2.8-7.0)$ & .344 \\
\hline
\end{tabular}

VASIbp-24 $h$ Visual Analogue Scale of Low Back Pain $24 \mathrm{~h}$ after Operation, VASlbp-48 $h$ Visual Analogue Scale of Low Back Pain $48 \mathrm{~h}$ after Operation, VASlbp-72 $h$ Visual Analogue Scale of Low Back Pain $72 \mathrm{~h}$ after Operation, VASperipheral VAS during Peripheral Venipuncture, VASdural Visual Analogue Scale during Subarachnoid Puncture, $\triangle$ VAS Difference between VASperipheral and VASdural, VASdural-24 $h$ VAS of Back Puncture Point $24 \mathrm{~h}$ after Operation, VASdural-48 $h$ VAS of Back Puncture Point $48 \mathrm{~h}$ after Operation, VASdural-72 h VAS of Back Puncture Point $72 \mathrm{~h}$ after Operation, IQR Interquartile Range 
and stressed posture resulted in the postural pain primarily, enormous physical and physiological changes during pregnancy and after delivery such as lumbar lordosis, center of gravity rise and fall, and loss of abdominal muscle support that resulted in intense stretch on the lower back should be also considered [11]. Wang $\mathrm{CH}$ [23] concluded that postpartum LBP could be related to changes during pregnancy and not related to spinal anesthesia, and this might be the reason for the negative result from our study in LBP using either 22 or 27 gauge needles. A cross-sectional study, which is the largest study on pregnancy-related LBP in the literature, also showed that about 1 in 2 women have pregnancy-related LBP in any stage of pregnancy [9].

The risk of venous thromboembolism (VTE) in pregnancy was 5.7/10000 [24]. Guidelines for anticoagulant indications and management have been published by different national and international agencies to prevent venous thromboembolism during pregnancy [25-27]. Although anticoagulation therapy was given, maternal thrombophilias were still increased among these parturients in our research, some of whom had high D-Dimer, normal platelet count and coagulation time. A therapeutic anticoagulation window of $12 \mathrm{~h}$ was recommended during delivery when using LMWH as prophylactic [28]. The mean delay between the last injection and the need for anesthesia in our data was more than $12 \mathrm{~h}$. Aspirin was not recommended for drug withdrawal according to the guidelines. Nevertheless, puerperas in our medical center usually underwent a delay of about 6 days before delivery. The use of anticoagulant therapy during pregnancy should be cautious because of the potential for both fetal and maternal complications.

Furthermore, our study showed that aspirin was the most common anticoagulant medication used by pregnant population in our medical center. Aspirin is a preferred anticoagulant and is widely accepted by doctors and parturients in our medical center, occupying the largest proportion of usage. It is suggested that oral low-dose aspirin treatment can prevent the occurrence of preeclampsia, but there are complication of placental infarction [29]. Currently, based on epidemiological evidence, it is recommended to take low-dose aspirin daily as a preventive intervention for high-risk preeclampsia during pregnancy after 12 weeks of gestation [30]. The preference of aspirin usage in our medical center may be due to the prevention of fetal growth restriction and a reversible increase in uterine blood flow [31], whereas evidence on the use of LMWH from randomized control trials in both in vitro and in vivo studies remained inconsistent [32-36]. The use of heparin, including LMWH, was established for the prevention/improvement of thrombophlebitis in the pathological hypercoagulability during pregnancy [37]. Meanwhile, the dosage regimen of coagulant used in our medical center mainly focused on alone and duplex medications rather than triple combination. In women with antiphospholipid (aPL) syndrome with recurrent miscarriages, to improve blood circulation, guidelines recommend an anticoagulant therapy of oral low-dose aspirin combined with intravenous LMWH [37, 38]. Further prospective randomized controlled trials are required to determine the optimal maternal hypercoagulant treatment strategy. Although the beneficial effects of Salvia Miltiorrhiza on anticoagulation, vasodilation, reduction of oxidative stress, and lipid profiles have been put forwarded, studies on Salvia Miltiorrhiza in obstetrics were limited [39]. Morton JS et al. investigated the use of Salvia Miltiorrhiza did not affect placenta- related factors and blood pressure of pregnant women with pregnancy-induced hypertension [40]. Hence, further study on larger sample size is required.

\section{Limitation of the study}

Insufficient sample size was the main limitation of our research. Our study did not include twins and obese parturients due to their distinctive body sizes. Because of the difficulty in studying the aim of the study in a prospective way and ethical limits, no magnetic resonance imaging (MRI) was performed on any parturient to check spinal or intracranial hematoma. In addition, since PDPH and LBP were developed till 5 to 7 days after dural puncture, the restricted observation period due to short hospital stay and lack of long-term follow-up information remained as limitations. Besides, our study was a single center study and requires recruitment of more parturients, and monitors for longer follow-up period to obtain confirmatory conclusion and explore the cause and mechanism in future study.

\section{Conclusion}

In conclusion, the present study demonstrates that minimally invasive spinal anesthesia provided a lower VAS during subarachnoid puncture, a lower VAS of back puncture point $24 \mathrm{~h}$ after operation and a higher maternal satisfaction. Hence, it was considered to be a safe, reliable and reasonable approach for parturients undergoing cesarean section in maternal anticoagulation therapy with normal platelet count and coagulation time. As the date of delivery is difficult to predict, minimally invasive spinal anesthesia could be a potential solution for parturients taking anticoagulants to go through cesarean section successfully by avoiding general anesthesia and get the experience of joy of baby birth moment.

\section{Abbreviations}

aPL: Antiphospholipid; CSF: Cerebrospinal fluid; CSR: Cesarean section rate; ICU: Intensive care unit; INR: International normalized ratio; IQR: Interquartile range; LBP: Low back pain; LMWH: Low molecular weight heparin; MRI: Magnetic resonance imaging; PDPH: Postdural puncture headache; 
SD: Standard deviation; VASdural: Visual analogue scale during subarachnoid puncture; VASdural-24 h: Visual analogue scale of back puncture point $24 \mathrm{~h}$ after operation; VASdural-48 h: Visual analogue scale of back puncture point $48 \mathrm{~h}$ after operation; VASdural-72 h: Visual analogue scale of back puncture point $72 \mathrm{~h}$ after operation; VASIbp: Visual analogue scale of low back pain; VASPDPH: Visual analogue scale of postdural puncture headache;

VASperipheral: Visual analogue scale of during peripheral venipuncture; VASIbp-24 h: Visual analogue scale of low back pain $24 \mathrm{~h}$ after operation; VASlbp-48 h: Visual analogue scale of low back pain $48 \mathrm{~h}$ after operation; VASIbp- $72 \mathrm{~h}$ : Visual analogue scale of low back pain $72 \mathrm{~h}$ after operation; VTE Venous: Thromboembolism; WHO: World health organization; $\triangle V A S:$ Difference between visual analogue scale during peripheral venipuncture and visual analogue scale during subarachnoid puncture

\section{Acknowledgements}

All authors thank all the nursing staff, and patients who participated in this study.

\section{Funding}

This study was funded by National Natural Science Foundation of China (81701358). The funding was used for English editing of manuscript.

\section{Availability of data and materials}

The datasets used and analyzed during the current study are available from the corresponding author on reasonable request.

\section{Authors' contributions}

All authors (DH, LZ, JC, and JZ) made substantial contributions to the study conception and design, were involved in revising this manuscript critically, approved the final manuscript and agreed to be accountable for all aspects of the work. $L Z$ collected and analyzed the patient data and DH was a major contributor in writing the manuscript. JC and JZ took part in the coordination of this project. DH applied for funding. DH and LIZ contributed equally to this work.

\section{Ethics approval and consent to participate}

The Ethics Committee of Renji Hospital, School of Medicine, Shanghai Jiaotong University reviewed the study protocol and the ethical approval was granted in March 2016 (No. 2016-033). Written informed consents were obtained either from the patients or legal representatives.

\section{Consent for publication}

All included patients or their family members signed the informed consent form to report individual patient data. All authors have confirmed the manuscript and approved the publication of the manuscript.

\section{Competing interests}

No conflict of interest was declared by any of the authors.

\section{Publisher's Note}

Springer Nature remains neutral with regard to jurisdictional claims in published maps and institutional affiliations.

\section{Received: 11 August 2018 Accepted: 28 December 2018}

\section{Published online: 12 January 2019}

\section{References}

1. Lumbiganon P, Laopaiboon M, Gulmezoglu AM, Souza JP, Taneepanichskul S, Ruyan P, Attygalle DE, Shrestha N, Mori R, Nguyen DH, et al. Method of delivery and pregnancy outcomes in Asia: the WHO global survey on maternal and perinatal health 2007-08. Lancet. 2010;375(9713):490-9.

2. Appropriate technology for birth. Lancet. 1985;2(8452):436-7. https://doi. org/10.1016/S0140-6736(85)92750-3.

3. Li HT, Luo S, Trasande L, Hellerstein S, Kang C, Li JX, Zhang Y, Liu JM, Blustein J. Geographic variations and temporal trends in cesarean delivery rates in China, 2008-2014. JAMA. 2017;317(1):69-76.

4. Angle P, Tang SL, Thompson D, Szalai JP. Expectant management of postdural puncture headache increases hospital length of stay and emergency room visits. Can J Anaesth. 2005;52(4):397-402.

5. Kuczkowski KM. Post-dural puncture headache in the obstetric patient: an old problem. New solutions. Minerva Anestesiol. 2004;70(12):823-30.
6. Lowery S, Oliver A. Incidence of postdural puncture headache and backache following diagnostic/therapeutic lumbar puncture using a $22 \mathrm{G}$ cutting spinal needle, and after introduction of a $25 \mathrm{G}$ pencil point spinal needle. Paediatr Anaesth. 2008:18(3):230-4.

7. Choi PT, Galinski SE, Takeuchi L, Lucas S, Tamayo C, Jadad AR. PDPH is a common complication of neuraxial blockade in parturients: a meta-analysis of obstetrical studies. Can J Anaesth. 2003;50(5):460-9.

8. Mogren IM, Pohjanen Al. Low back pain and pelvic pain during pregnancy: prevalence and risk factors. Spine (Phila Pa 1976). 2005;30(8):983-91.

9. Sencan S, Ozcan-Eksi EE, Cuce I, Guzel S, Erdem B. Pregnancy-related low back pain in women in Turkey: prevalence and risk factors. Ann Phys Rehabil Med. 2018;61(1):33-7.

10. Turgut F, Turgut $M$, Cetinsahin M. A prospective study of persistent back pain after pregnancy. Eur J Obstet Gynecol Reprod Biol. 1998;80(1):45-8.

11. Chia YY, Lo Y, Chen YB, Liu CP, Huang WC, Wen CH. Risk of chronic low Back pain among Parturients who undergo cesarean delivery with Neuraxial anesthesia: a Nationwide population-based retrospective cohort study. Medicine (Baltimore). 2016;95(16):e3468.

12. Chow L, Farber MK, Camann WR. Anesthesia in the pregnant patient with hematologic disorders. Hematol Oncol Clin North Am. 2011;25(2):425-43 ix-x.

13. Pan $\mathrm{PH}$, Fragneto $\mathrm{R}$, Moore $\mathrm{C}$, Ross $\mathrm{V}$. Incidence of postdural puncture headache and backache, and success rate of dural puncture: comparison of two spinal needle designs. South Med J. 2004;97(4):359-63.

14. Headache Classification Committee of the International Headache S. The international classification of headache disorders, 3rd edition (beta version). Cephalalgia. 2013;33(9):629-808.

15. Gogarten W, Vandermeulen E, Van Aken H, Kozek S, Llau JV, Samama CM. European Scoeity of a: regional anaesthesia and antithrombotic agents: recommendations of the European society of Anaesthesiology. Eur J Anaesthesiol. 2010;27(12):999-1015.

16. Nathan N, Sol I, Vincelot A, Collet D, Granchamp P, David E. Is a biological assessment necessary to realize an obstetrical epidural anaesthesia in a patient whose interrogation and clinical examination are strictly normal? Ann Fr Anesth Reanim. 2007;26(7-8):705-10.

17. Horlocker TT, Wedel DJ, Rowlingson JC, Enneking FK, Kopp SL, Benzon HT, Brown DL, Heit JA, Mulroy MF, Rosenquist RW, et al. Regional anesthesia in the patient receiving antithrombotic or thrombolytic therapy: American Society of Regional Anesthesia and Pain Medicine evidence-based guidelines (third edition). Reg Anesth Pain Med. 2010;35(1):64-101.

18. Kim M, Yoon H. Comparison of post-dural puncture headache and low back pain between 23 and 25 gauge Quincke spinal needles in patients over 60 years: randomized, double-blind controlled trial. Int J Nurs Stud. 2011;48(11):1315-22.

19. Lambert DH, Hurley RJ, Hertwig L, Datta S. Role of needle gauge and tip configuration in the production of lumbar puncture headache. Reg Anesth. 1997;22(1):66-72.

20. Mylius V, Kunz M, Hennighausen E, Lautenbacher S, Schepelmann K. Effects of ageing on spinal motor and autonomic pain responses. Neurosci Lett. 2008;446(2-3):129-32.

21. Lautenbacher S, Kunz M, Strate P, Nielsen J, Arendt-Nielsen L. Age effects on pain thresholds, temporal summation and spatial summation of heat and pressure pain. Pain. 2005;115(3):410-8.

22. Yee K, Shetty AL, Lai K. ABG needle study: a randomised control study comparing $23 \mathrm{G}$ versus $25 \mathrm{G}$ needle success and pain scores. Emerg Med J. 2015;32(5):343-7.

23. Wang $\mathrm{CH}$, Cheng KW, Neoh CA, Tang S, Jawan B, Lee JH. Comparison of the incidence of postpartum low back pain in natural childbirth and cesarean section with spinal anesthesia. Acta Anaesthesiol Sin. 1994;32(4):243-6.

24. Parunov LA, Soshitova NP, Ovanesov MV, Panteleev MA, Serebriyskiy II. Epidemiology of venous thromboembolism (VTE) associated with pregnancy. Birth Defects Res C Embryo Today. 2015;105(3):167-84.

25. Benhamou D, Mignon A, Aya G, Brichant JF, Bonnin M, Chauleur C, Deruelle P, Ducloy AS, Edelman P, Rigouzzo A, et al. Prophylaxis of thromboembolic complications in obstetrics and gynaecology. Ann Fr Anesth Reanim. 2005; 24(8):911-20.

26. Bates SM, Greer IA, Middeldorp S, Veenstra DL, Prabulos AM, Vandvik PO. VTE, thrombophilia, antithrombotic therapy, and pregnancy: antithrombotic therapy and prevention of thrombosis, 9th ed: American College of Chest Physicians Evidence-Based Clinical Practice Guidelines. Chest. 2012;141(2 Suppl):e691S-736S.

27. Saar JA, Maack C. European Society of C: [diagnosis and management of acute pulmonary embolism. ESC guidelines 2014]. Herz. 2015;40(8):1048-54. 
28. Roueli A, Cesario E, Amsellem J, Agman A, Vauthier-Brouzes D, Nizard J. Is a therapeutic anticoagulation window needed for delivery when using prophylactic low molecular weight heparin during pregnancy? A retrospective monocentric study. Eur J Obstet Gynecol Reprod Biol. 2017; 215:118-23.

29. Suzuki Y, Hattori T, Kajikuri J, Yamamoto T, Suzumori K, Itoh T. Reduced function of endothelial prostacyclin in human omental resistance arteries in pre-eclampsia. J Physiol. 2002;545(Pt 1):269-77.

30. Osikoya O, Jaini PA, Nguyen A, Valdes M, Goulopoulou S. Effects of low-dose aspirin on maternal blood pressure and vascular function in an experimental model of gestational hypertension. Pharmacol Res. 2017;120:267-78.

31. Kang X, Wang T, He L, Xu H, Liu Z, Zhao A. Effect of low-dose aspirin on Midluteal phase uterine artery blood flow in patients with recurrent pregnancy loss. J Ultrasound Med. 2016;35(12):2583-7.

32. Groom KM, David AL. The role of aspirin, heparin, and other interventions in the prevention and treatment of fetal growth restriction. Am J Obstet Gynecol. 2018;218(2S):S829-40.

33. Bose P, Black S, Kadyrov M, Bartz C, Shlebak A, Regan L, Huppertz B. Adverse effects of lupus anticoagulant positive blood sera on placental viability can be prevented by heparin in vitro. Am J Obstet Gynecol. 2004;191(6):2125-31.

34. Di Simone N, Di Nicuolo F, Sanguinetti M, Ferrazzani S, D'Alessio MC, Castellani R, Bompiani A, Caruso A. Low-molecular weight heparin induces in vitro trophoblast invasiveness: role of matrix metalloproteinases and tissue inhibitors. Placenta. 2007;28(4):298-304.

35. Ganapathy R, Whitley GS, Cartwright JE, Dash PR, Thilaganathan B. Effect of heparin and fractionated heparin on trophoblast invasion. Hum Reprod. 2007;22(9):2523-7.

36. Quenby S, Mountfield S, Cartwright JE, Whitley GS, Vince G. Effects of lowmolecular-weight and unfractionated heparin on trophoblast function. Obstet Gynecol. 2004;104(2):354-61.

37. Makino A, Suzuki Y, Yamamoto T, Sato T, Kojima K, Suzumori K. Use of aspirin and low-molecular-weight heparin to prevent recurrence of maternal floor infarction in women without evidence of antiphospholipid antibody syndrome. Fetal Diagn Ther. 2004;19(3):261-5.

38. Middeldorp S. Anticoagulation in pregnancy complications. Hematology Am Soc Hematol Educ Program. 2014:2014(1):393-9.

39. Morton JS, Abdalvand A, Jiang Y, Sawamura T, Uwiera RR, Davidge ST. Lectin-like oxidized low-density lipoprotein 1 receptor in a reduced uteroplacental perfusion pressure rat model of preeclampsia. Hypertension. 2012;59(5):1014-20.

40. Morton JS, Quon A, Cheung PY, Sawamura T, Davidge ST. Effect of sodium tanshinone IIA sulfonate treatment in a rat model of preeclampsia. Am J Physiol Regul Integr Comp Physiol. 2015;308(3):R163-72.

Ready to submit your research? Choose BMC and benefit from:

- fast, convenient online submission

- thorough peer review by experienced researchers in your field

- rapid publication on acceptance

- support for research data, including large and complex data types

- gold Open Access which fosters wider collaboration and increased citations

- maximum visibility for your research: over $100 \mathrm{M}$ website views per year

At $\mathrm{BMC}$, research is always in progress.

Learn more biomedcentral.com/submissions 